\title{
Network-Based Analysis Reveals Functional Connectivity Related to Internet Addiction Tendency
}

\author{
Tanya Wen ${ }^{1,2 *}$ and Shulan Hsieh ${ }^{1,3,4 *}$ \\ ${ }^{1}$ Cognitive Electrophysiology Lab: Control, Aging, Sleep, and Emotion, Department of Psychology, National Cheng Kung \\ University, Tainan, Taiwan, ${ }^{2}$ Department of Life Sciences, National Cheng Kung University, Tainan, Taiwan, ${ }^{3}$ Institute of Allied \\ Health Sciences, National Cheng Kung University, Tainan, Taiwan, ${ }^{4}$ Department of Public Health, National Cheng Kung \\ University, Tainan, Taiwan
}

OPEN ACCESS

Edited by:

Chiang-shan R. Li,

Yale University, USA

Reviewed by:

Xin Di,

New Jersey Institute of Technology,

USA

Jintao Zhang,

Beijing Normal University, China

*Correspondence:

Shulan Hsieh

psyhsı@mail.ncku.edu.tw;

Tanya Wen

tanya.wen@mrc-cbu.cam.ac.uk

Received: 16 September 2015

Accepted: 11 January 2016

Published: 01 February 2016

Citation:

Wen $T$ and Hsieh S (2016) Network-Based Analysis Reveals Functional Connectivity Related to Internet Addiction Tendency.

Front. Hum. Neurosci. 10:6. doi: 10.3389/fnhum.2016.00006
Preoccupation and compulsive use of the internet can have negative psychological effects, such that it is increasingly being recognized as a mental disorder. The present study employed network-based statistics to explore how whole-brain functional connections at rest is related to the extent of individual's level of internet addiction, indexed by a self-rated questionnaire. We identified two topologically significant networks, one with connections that are positively correlated with internet addiction tendency, and one with connections negatively correlated with internet addiction tendency. The two networks are interconnected mostly at frontal regions, which might reflect alterations in the frontal region for different aspects of cognitive control (i.e., for control of internet usage and gaming skills). Next, we categorized the brain into several large regional subgroupings, and found that the majority of proportions of connections in the two networks correspond to the cerebellar model of addiction which encompasses the four-circuit model. Lastly, we observed that the brain regions with the most inter-regional connections associated with internet addiction tendency replicate those often seen in addiction literature, and is corroborated by our meta-analysis of internet addiction studies. This research provides a better understanding of large-scale networks involved in internet addiction tendency and shows that pre-clinical levels of internet addiction are associated with similar regions and connections as clinical cases of addiction.

Keywords: internet addiction, network-based statistics, functional connectivity, resting-state, meta-analysis

\section{INTRODUCTION}

Internet addiction (OReilly, 1996; Young, 1998) is a modern phenomenon that is characterized by preoccupation and compulsive use of the internet. In particular, internet gaming disorder (IGD) has been listed in Section III of the Diagnostic and Statistical Manual version 5 (DSM$5^{\circledR}$, American Psychiatric Association [APA], 2013). Due to a lack of a standard criterion, some literature treated the two terminology as synonymous (see Petry and O'Brien, 2013; Petry et al., 2014 for a discussion); however, the compulsive and excessive use of the internet for any activity (which we will refer to in this literature as internet addiction) is more global than its major subtype IGD, which can include multiple forms of internet use in addition to online gaming (Griffiths and Pontes, 2014; Király et al., 2014; Petry et al., 2014). Our current study investigates internet addiction 
in the more general form. Similar to substance use disorders, internet addiction shows withdrawal symptoms, tolerance, loss of control, and psychosocial problems, leading to clinically significant distress or impairment in daily functioning. Prevalence seems highest Asian countries and in male adolescents, and has been estimated to range from 14.1 to 16.5\% (95 percent confidence interval) among Taiwan college students in one study (Lin et al., 2011). The phenomenon has been attracting more attention over the past few years and clearly deserves further research.

Functional magnetic resonance imaging (fMRI) has been employed to identify the neural substrates of internet addiction, which turned out to show similar brain signatures with substance-related addictions (Kuss and Griffiths, 2012; Brand et al., 2014; Meng et al., 2015). In blocked and event-related studies, several regions associated with reward, addiction, and craving have been identified by contrasting internet gaming cues with baseline, which includes the insula, nucleus accumbens (NAc), dorsolateral prefrontal cortex (DLPFC), and orbital frontal cortex (OFC) (Hoeft et al., 2008; Ko et al., 2009; Han et al., 2010; Sun et al., 2012; Ko et al., 2013). However, activation-based approaches contrast cue-related activity and do not address how regions of the brain interact, and thus cannot characterize altered functional connections associated with clinical or behavioral measures; yet human disorders are a result of disturbances in an interconnected complex system (Fornito and Bullmore, 2015). The introduction of resting-state fMRI has proved to be a powerful tool for studying whole brain neural connectivity (van den Heuvel and Pol, 2010). Resting-state functional connectivity is assessed by the correlation of spontaneous fluctuations of blood oxygen level-dependent (BOLD) signals in different regions of the brain and is thought to provide a measure of its functional organization, and can help characterize abnormal synchronizations between brain regions in the spectrum of psychological phenotypes (Biswal et al., 2010; Craddock et al., 2013).

Although there have been some studies that have employed functional connectivity to investigate altered functional connectivity associated with internet addiction, most studies used seed regions chosen a priori, either (a) correlating one seed region with the remaining voxels of the whole brain [Hoeft et al., 2008 used the NAc; Lorenz et al., 2013 used the right inferior frontal gyrus (IFG); Ding et al., 2013 used the posterior cingulate cortex (PCC); Ko et al., 2015 used the amygdala; Zhang et al., 2015 used the insula; Hong et al., 2015 used the caudate nucleus and putamen; Kühn and Gallinat, 2015 used the right frontal pole; Li et al., 2015 used the right DLPFC] or (b) performing correlations among multiple predefined ROIs selected from meaningful networks (Yuan et al., 2015 examined the central executive network and salience network; Dong et al., 2015b examined the executive control network; Dong et al., 2015a examined the executive control network and reward network; Li et al., 2014 examined the response inhibition network; Lin et al., 2015 examined six predefined bilateral corticostriatal ROIs). The pre-defined seed regions examined only represent a small proportion of the brain, thus they may not be able to provide a complete picture of how the connectome is affected by internet addiction.

Very few studies have used a whole-brain approach to study internet addiction. To our knowledge, there are currently only four published papers that adopted a whole-brain approach, and their methods are quite variable, ranging from network-based statistics (NBS; Hong et al., 2013) to topological (Hong et al., 2013; Wee et al., 2014; Park et al., 2015) to a novelly developed voxel-mirrored homotopic connectivity (Wang et al., 2015). In particular, Hong et al. (2013) employed NBS to identify betweengroup differences in inter-regional functional connectivity, and found impaired connections involved in cortico-subcortical circuits in patients with internet addiction. However, their study focused on a small sample size of a unique population (male early adolescents).

Therefore, in our current paper, we decided to use a wholebrain connectivity approach, NBS (Zalesky et al., 2010a; Han et al., 2013), to identify functional connections that are predictive of internet addiction tendency. NBS is a validated statistical method to deal with the multiple comparisons problem on a graph, it is analogous to cluster-based methods (Nichols and Holmes, 2002), and is used to identify connections and networks comprising the human connectome that are associated with an experimental effect or a between-group difference by testing the hypothesis independently at every connection. Our results will furthermore be compared with a meta-analysis of existing papers related to the neural correlates of internet addiction. We hope to extend the existing literature in several ways: (1) We hope to provide a more complete picture of internet addiction by using whole-brain analysis instead of using only a small number of pre-defined seed regions. (2) Although there exists a couple of whole-brain functional connectivity studies on internet addiction (e.g., Hong et al., 2013; Wang et al., 2015), the studies compared internet addiction groups with healthy controls. Our study did not involve any clinical patients, but characterized internet addiction tendency as a gradient. We hope to identify functional connections whose strength is modulated by level of addiction. (3) Most internet addiction studies have not taken the cerebellum into consideration, yet the cerebellum has been implicated as an important region in addiction (Moulton et al., 2014). Thus, we have included the cerebellum in our analysis. (4) Many studies have limited their participant group to males, and often contain relatively small sample sizes (e.g., Hong et al., 2013, 2015; Kühn and Gallinat, 2015). To increase the generalizability and power of these studies, samples containing both genders and a larger sample size is necessary (Li et al., 2015). By tackling the above problems, the current study hopes to provide a better understanding of how functional connectivity is associated with internet addiction tendency.

\section{MATERIALS AND METHODS}

\section{Meta-Analysis}

A meta-analysis was constructed using the NeuroSynth database (http://neurosynth.org; Yarkoni et al., 2011). A customized 
analysis was performed by using the search terms "addiction," "addict," "internet," "gaming," "game," and "online" to identify studies related to internet addiction in the database. The criteria of inclusion was verified manually, and a list of the studies included are detailed in the Supplementary Materials 1. A total of 18 studies were included. Peak activation coordinates as well as its neighborhood of $6 \mathrm{~mm}$ voxels were extracted from the included studies. Next, a meta-analysis of these coordinates was performed, producing forward and revere inference whole-brain $z$-score maps. The forward inference maps reflect the likelihood that a region will activate given these terms $[P$ (activation| terms)], therefore informing us of the consistency of activation for the given terms. The reverse inference map shows the likelihood that these terms are used in a study given the presence of reported activation $[P($ terms $\mid$ activation)]; thus a region that is activated indicates it is more likely to be an internet addiction related study than a non-internet addiction related study, reflecting selectivity of that region. Since both forward and reverse inference play an important role in helping us understand regions associated with internet addiction, we overlapped these two inference maps to outline their common regions. Clusters greater than five voxels are reported.

\section{Resting-State fMRI Participants}

Forty-seven healthy participants (21 males and 26 females) from southern Taiwan, most of which are students or staff in the university, were recruited through advertisements, to participate in the experiment (age range $=19-29$ years, mean age $=22.87$ years, $S D=2.22$ years). The participants were righthanded (indicated by the Edinburgh Handedness Inventory), had normal or corrected-to-normal vision, and no history of psychological or neural disorders. Their depression, anxiety and intelligence scores were in the normal range [Beck's Depression Inventory (BDI) score: 0-12; Beck's Anxiety Inventory (BAI) score: 0-7; Raven's Standard Progressive Matrices test score: 35-57]. The Chen Internet Addiction Scale-Revised (CIAS-R) score of all participants had range $=28-92$, mean $=60.04$, $S D=16.53$. Table 1 summarizes the demographic information and behavioral characteristics of the participants. The normality of the CIAS-R scores was verified by the Shapiro-Wilk test $[W(47)=0.98, p=0.50]$. There was no significant correlation between gender and CIAS-R score (Spearman's $\rho=0.15$,

TABLE 1 | Demographic information and behavioral characteristics.

\begin{tabular}{lcrr}
\hline & Range & Mean & SD \\
\hline Age & $19-29$ & 22.87 & 2.22 \\
BDI & $0-12$ & 4.17 & 3.74 \\
BAl & $0-7$ & 1.81 & 2.02 \\
SPM & $35-57$ & 46.28 & 5.19 \\
CIAS-R & $28-92$ & 60.04 & 16.53 \\
\hline
\end{tabular}

${ }^{*} B D I$, Beck's Depression Inventory; BAI, Beck,s Anxiety Inventory; SPM, Raven's Standard Progressive Matrices; CIAS-R, Chen Internet Addiction Scale-Revised. $p=0.30)$. All participants provided their written informed consent, and the study protocol was approved (NO: B-ER101-144) by the Institutional Review Board (IRB) of the National Cheng Kung University Hospital, Tainan, Taiwan. All participants were paid 500 NTD after completion of the experiment.

\section{Chen Internet Addiction Scale-Revised (CIAS-R) Questionnaire}

The Chen Internet Addiction Scale-Revised (CIAS-R; Chen et al., 2003) is a 26-item measure used to assess the severity of internet addiction. The CIAS-R is based on the DSM-IV-TR additive behaviors criteria and contains two subscales of internet addiction (a) core symptoms and (b) related problems, assessing five dimensions including (1) compulsive internet use, (2) withdrawal symptoms when the internet is taken away, (3) tolerance, (4) jeopardy of interpersonal relationships and physical health, and (5) time management problems. Items are rated on a 4-point Likert scale, with total scores ranging from 26 to 104, reflecting low to high tendency of internet addiction. It has been shown that the CIAS-R has high internal consistency (Cronbach's $\alpha=0.79-0.93$; Chen et al., 2003) and high diagnostic accuracy (AUC $=89.6 \%$; Ko et al., 2005). In the present study, the CIAS-R total score was utilized as an indicator of participants' current status of internet addiction.

\section{Image Acquisition and Processing}

Imaging was performed using the GE MR750 3T scanner (GE Healthcare, Waukesha, WI, USA) in the MRI center of National Cheng Kung University. High resolution anatomical images were acquired using fast-SPGR, consisting of 166 axial slices $\left(T R=7.6 \mathrm{~ms}, T E=3.3 \mathrm{~ms}\right.$, flip angle $171=12^{\circ}$, 224 matrices $\times 224$ matrices, slice thickness $=1 \mathrm{~mm}$ ). Functional images were acquired using a gradient-echo echo-planar imaging (EPI) pulse sequence $(T R=2000 \mathrm{~ms}, T E=30 \mathrm{~ms}$, flip angle $=77^{\circ}, 64$ matrices $\times 64$ matrices, slice thickness $=4 \mathrm{~mm}$, no gap, voxel size $3.4375 \mathrm{~mm} \times 3.4375 \mathrm{~mm} \times 4 \mathrm{~mm}, 32$ axial slices covering the entire brain).

Participants were told to relax and lie in the scanner with their eyes closed. They were asked not to think about any particular event while scanning. The scanning time for the structural image was approximately $3.6 \mathrm{~min}$. The functional image lasted approximately $8 \mathrm{~min}$, with the first five TRs serving as dummy scans to ensure that the signal has reached a steady state before data are collected; thus a run consists of 240 EPI volume images for analysis.

The data was preprocessed using the Data Processing Assistant for Resting-State fMRI (DPARSF; Yan and Zang, 2010), which is based on functions in MRIcroN (Rorden et al., 2007 $)$ as well as Statistical Parametric Mapping software $\left(\mathrm{SPM}^{2}\right)$ and the Resting-State fMRI Data Analysis Toolkit (REST; Song et al., 2011) in Matlab (The MathWorks, Inc., Natick, MA, USA). Functional images undergone slice timing correction,

\footnotetext{
${ }^{1}$ http://www.mricro.com

${ }^{2}$ http://www.fil.ion.ucl.ac.uk/spm
} 

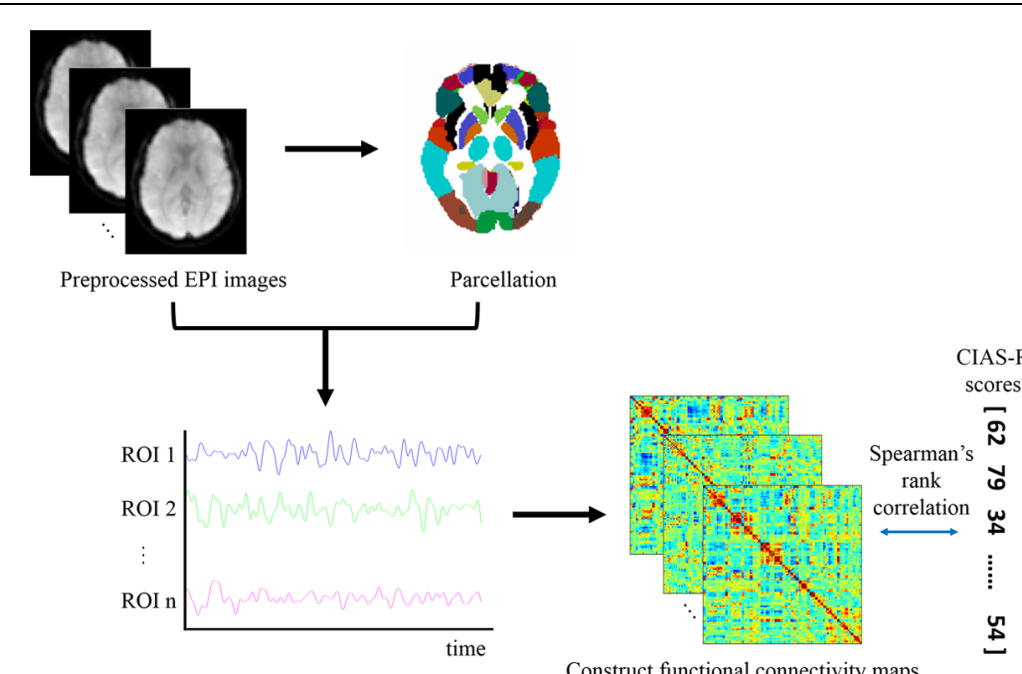

Time course extraction

Construct functional connectivity map
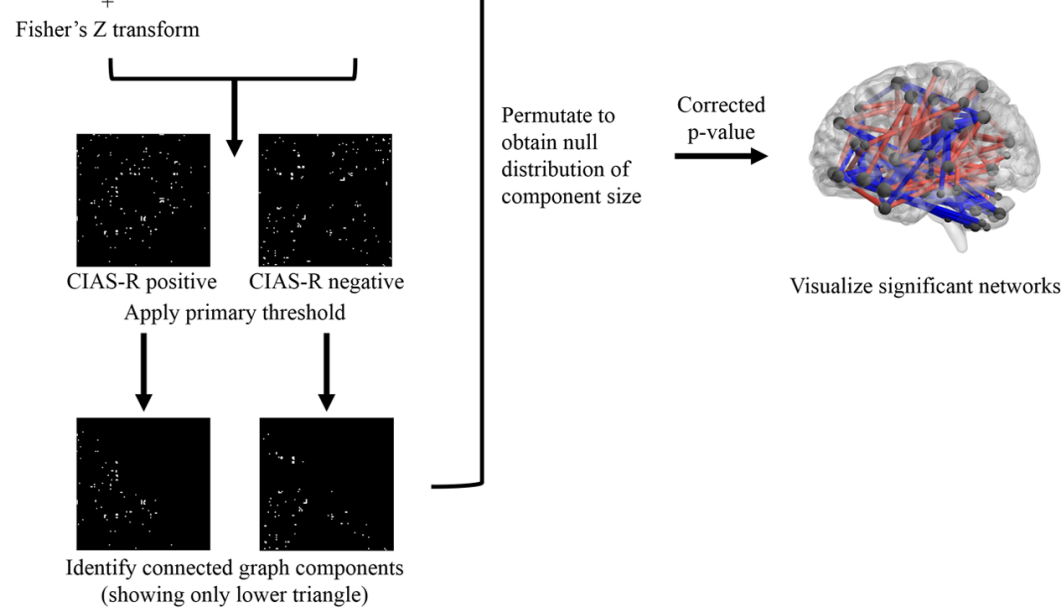

FIGURE 1 | Flowchart of data analysis pipeline. Participants' brains were preprocessed and parcellated to different structural regions according to the AAL template. A correlation matrix was constructed using the time courses extracted from each region to characterize connectivity between each pair of brain region. Network-based statistics was used to identify significant networks related to internet addiction tendency indexed by the CIAS-R score, which included three steps: (1) mass univariate testing of Spearman's rank correlation of each cell of the correlation matrix and CIAS-R scores, (2) application of primary threshold to select for highly correlated connections, and (3) identify the largest number of connected graph components. The preceding three steps were permutated to obtain a null distribution of the largest component size, and further used to test the significance of the identified network(s).

followed by realignment to correct for head motion using six-parameter rigid body transformations. The overall motion, characterized by mean framewise displacement (FD), was not large $($ mean $=0.05, S D=0.03$ ) and did not correlate with the CIAS-R scores (Spearman's $\rho=-0.28, p=0.055$ ), thus impulsivity is not a confounding factor of internet addiction score and motion (Kong et al., 2014). T1 images were coregistered to functional images. Structural images were segmented into CSF, white matter and gray matter based on tissue probability maps in MNI space, and these calculations were used in the subsequent normalization of T1 and EPI images to MNI space. The data were smoothed in the spatial domain using a Gaussian kernel of $6 \mathrm{~mm}$ full width at half maximum (FWHM) and removed of linear trend. Nuisance covariates including global mean signal, white matter signal, and cerebrospinal fluid signal were regressed out. Although whether to perform the global signal regression is still an ongoing controversy (e.g., Saad et al., 2012), we decided to implement this method because it has been suggested to maximize the specificity of functional correlations and improve the correspondence between restingstate correlations and anatomy (Fox et al., 2009; Weissenbacher et al., 2009; Takeuchi et al., 2014). Finally, the images undergone band-pass filtering of $0.01-0.08 \mathrm{~Hz}$.

\section{Data Analysis}

The fMRI images were parcellated based on the Anatomical Automatic Labeling (AAL; Tzourio-Mazoyer et al., 2002) template, dividing the brain based on anatomical structure into 116 ROIs (or nodes). We chose the AAL atlas because it has been the most commonly used parcellation in functional network studies (Stanley et al., 2013) and was also the template used by Hong et al. (2013), whose study is most relevant to ours, thus 
increasing the degree of comparability across studies (Zalesky et al., 2010b). The NBS method was used to identify brain networks that consists of inter-regional functional connectivity showing significant correlation with CIAS-R score. The following analyses was done with the aid of the Network Based Statistic Toolbox (Zalesky et al., 2010a) with additional in-house Matlab scripts. A $116 \times 116$ correlation matrix was constructed for each participant using the time courses extracted from each ROI. The Pearson's $r$ values were normalized to $Z$ scores using Fisher's $Z$ transformation. Each cell of the correlation matrix represent the strength of the connection (or edge) between two nodes. Mass univariate testing using Spearman's rank correlation was performed between participants' CIAS-R scores and edge strengths within each edge to identify relevant connections that were predictive of the CIAS-R score. Candidate edges that showed high predictability of CIAS-R score were selected via a primary threshold of Spearman's rho $>0.37$ and $<-0.37$ (approximately the one-tailed alpha $=0.005$ ) respectively, to identify networks that are positively and negatively associated with CIAS-R score. Next, topological clusters, known as connected graph components were identified among the suprathreshold connections. A familywise error (FWE) for the component size was calculated using permutation testing (3000 permutations), which involved randomly reordering the CIAS-R scores and repeating the above process each permutation to obtain a null distribution of the largest component size. Connected graph components whose size exceeds the estimated FWE-corrected $p$-value cutoff of $<0.05$ were identified as networks that are significantly related to internet addiction tendency. BrainNet Viewer (Xia et al., 2013) was used for the visualization of connections. An illustration of the data analysis pipeline is shown in Figure 1.

\section{RESULTS}

\section{Meta-Analysis}

Forward and reverse inference $z$-score maps were generated from NeuroSynth (shown in Figure 2). The activations in these two maps show high resemblance of each other. Overlapping these maps revealed activation in regions of the cerebellum, temporal lobe (bilateral inferior temporal gyri, right superior temporal pole, and right middle and superior temporal gyrus), several frontal regions (left middle and superior orbital frontal gyrus, right middle frontal gyrus, right inferior frontal operculum, and right precentral gyrus), bilateral putamen, bilateral insula, right middle cingulate, and right precuneus. Table 2 lists the clusters identified as well as AAL regions belonging to the cluster.

\section{Resting-State fMRI \\ Functional Connections Related to Internet Addiction Tendency}

Using NBS, we identified two networks that showed significant correlation of edge strength and CIAS-R scores $(p<0.05$, FWEcorrected): one with edges positively correlated with CIAS-R scores ("CIAS-R positive," shown in red), and one with edges negatively correlated with CIAS-R (“CIAS-R negative," shown in blue). The CIAS-R positive network consists a total of 65 nodes and 90 edges (45 intrahemispheric, 42 interhemispheric, and 3 connecting to the vermis), while the negative network consist of 64 nodes and 89 edges ( 35 intrahemispheric, 40 interhemispheric, and 14 connecting to/within the vermis). It is important to note that the two networks are not completely separate, and they share a total of 39 nodes, $30.77 \%$ of which are frontal lobe regions. The total number of edges related to CIAS-R consist of $2.68 \%$

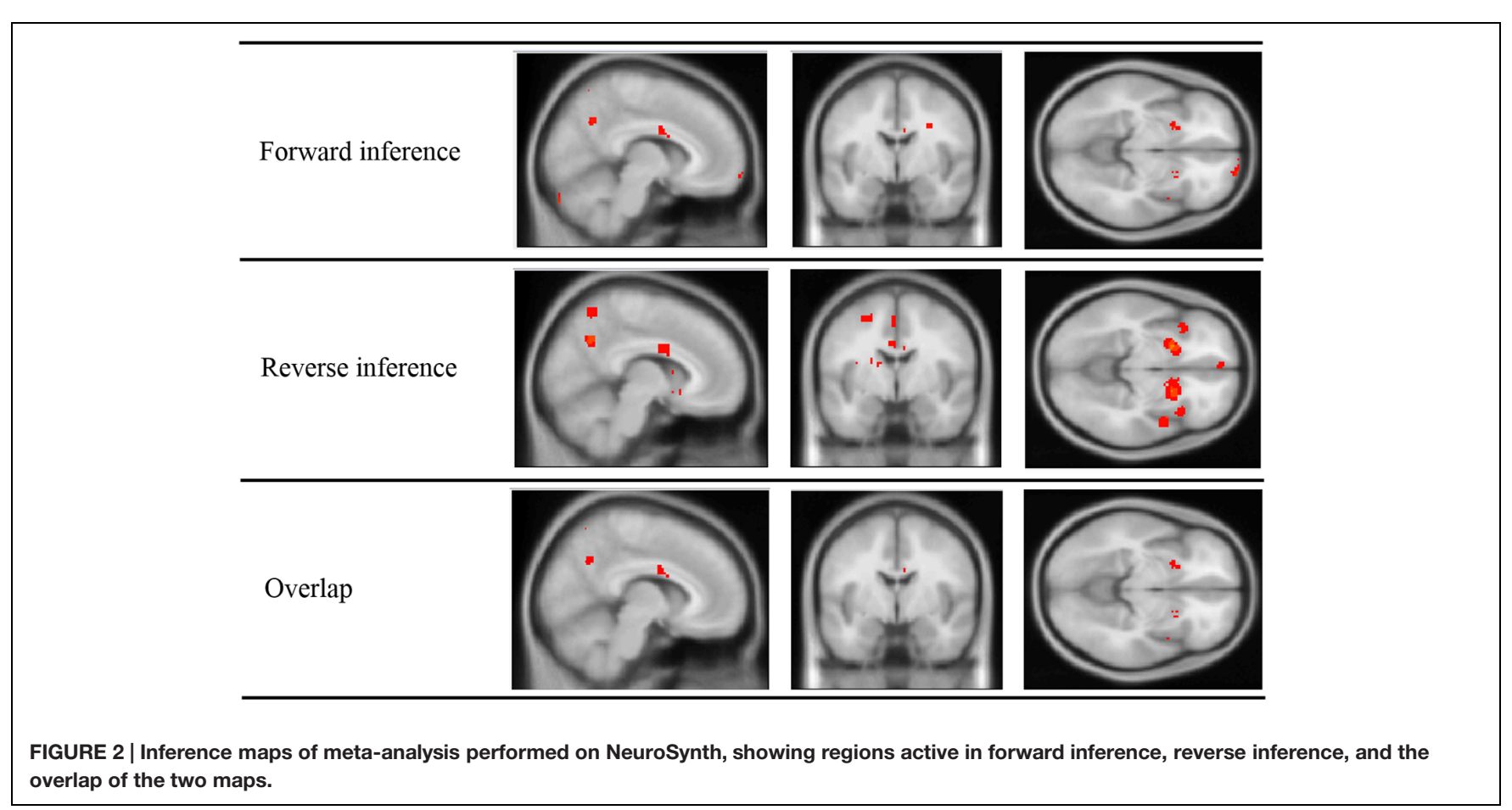


of all edges of the brain. The network is illustrated in Figure 3 and specific connections are listed in Supplementary Materials 2, Table S1.

\section{Global Distribution of Involved Edges}

To get a better understanding of how these connections are distributed, we followed Fornito et al. (2011) and Hong et al. (2013), and categorized each AAL region within each network as belonging to seven regional subgroupings: frontal, temporal, parietal, occipital, insula and cingulate gyri, subcortical, and cerebellum. The majority of edges in the CIAS-R positive network involved connections between (1) temporal regions and insula and cingulate gryi $(\sim 13 \%)$, most of which involves the posterior cingulate gyrus connecting to various temporal regions; (2) frontal and temporal regions $(\sim 12 \%)$, which includes connections between the medial orbitofrontal cortex, paracentral lobule and the temporal lobe gyri, temporal pole; and (3) parietal and subcortical regions $(\sim 11 \%)$, consisting of connections between the postcentral cortex and superior parietal lobule with the putamen and pallidum. It is interesting to note that except for the frontal lobe, all others regions do not have any intraregional connections whose strength is positively correlated with internet addiction tendency. The majority of edges in the CIAS-R negative network involved connections between (1) the frontal lobe and cerebellum $(\sim 19 \%)$, most of which are connections between the orbital frontal regions and various ROIs of the cerebellum; and (2) insula and cingulate gyri and the temporal lobe $(\sim 12 \%)$, which comprises connections between the insula, cingulum, parahippocampal, and temporal lobe gyri. No occipital regions were found to be included in the CIAS-R negative network. The proportions of inter-regional connections of each network is illustrated in Figure 4.

\section{Maximally Affected Nodes}

Due to the large number of edges identified, we followed Finn et al. (2014), and identified nodes that have a high "sum of CIAS-R-correlated edges" in order to focus our analysis on regions where connections are maximally related to internet addiction tendency. The sum of CIAS-R-correlated edges of a node was defined as the total number of its edges in both CIAS$\mathrm{R}$ positive and CIAS-R negative networks (this is conceptually equivalent to the degree measure in graph theory). This method will enable us to identify nodes where connections are most likely to be altered by internet addiction tendency. The following Table 3 lists the nodes that are maximally affected, and shows nodes that have at least a sum of CIAS-R-correlated edges of at least 8. Visualization of the nodes and their connections is displayed in Figure 5. These are also the nodes selected for discussion.

\section{DISCUSSION}

In a normal group of young adults, we assessed their level of internet addiction through a self-rated questionnaire (CIAS-R), and further identified two brain networks of which functional connections correlated positively and negatively with internet
TABLE 2 | Overlapping clusters of forward and reverse inference maps.

\begin{tabular}{|c|c|c|c|c|c|}
\hline \multirow{2}{*}{$\begin{array}{l}\text { Regions } \\
\text { Cluster } 1\end{array}$} & & \multirow{2}{*}{$\begin{array}{c}\begin{array}{c}\text { \# of voxels } \\
\left(\mathbf{2}^{*} \mathbf{2}^{*} \mathbf{2}\right)\end{array} \\
194\end{array}$} & \multicolumn{3}{|c|}{ Peak MNI co-ordinates } \\
\hline & & & -12 & -78 & -46 \\
\hline \multicolumn{6}{|c|}{ Cerebelum_Crus2_L } \\
\hline & \multicolumn{5}{|l|}{ Cerebelum_7b_L } \\
\hline & \multicolumn{5}{|l|}{ Cerebelum_Crus1_L } \\
\hline & \multicolumn{5}{|l|}{ Vermis_8 } \\
\hline & \multicolumn{5}{|l|}{ Cerebelum_8_L } \\
\hline & \multicolumn{5}{|l|}{ Vermis_7 } \\
\hline \multirow[t]{4}{*}{ Cluster 2} & & 70 & -22 & -78 & -52 \\
\hline & \multicolumn{5}{|l|}{ Cerebelum_Crus2_L } \\
\hline & \multicolumn{5}{|l|}{ Cerebelum_7b_L } \\
\hline & \multicolumn{5}{|l|}{ Cerebelum_8_L } \\
\hline \multirow[t]{2}{*}{ Cluster 3} & & 36 & 10 & 0 & 24 \\
\hline & \multicolumn{5}{|l|}{ Cingulum_Mid_R } \\
\hline \multirow[t]{3}{*}{ Cluster 4} & & 30 & -22 & 52 & -18 \\
\hline & \multicolumn{5}{|l|}{ Frontal_Mid_Orb_L } \\
\hline & \multicolumn{5}{|l|}{ Frontal_Sup_Orb_L } \\
\hline \multirow[t]{2}{*}{ Cluster 5} & & 26 & 10 & -62 & 34 \\
\hline & \multicolumn{5}{|l|}{ Precuneus_R } \\
\hline \multirow[t]{2}{*}{ Cluster 6} & & 20 & 52 & -50 & -26 \\
\hline & \multicolumn{5}{|l|}{ Temporal_Inf_R } \\
\hline \multirow[t]{2}{*}{ Cluster 7} & & 19 & -24 & 8 & -10 \\
\hline & Putamen_L & & & & \\
\hline Cluster 8 & & 17 & 20 & 10 & -10 \\
\hline & Putamen_R & & & & \\
\hline Cluster 9 & & 17 & 38 & 2 & 34 \\
\hline & Frontal_Mid_R & & & & \\
\hline & Precentral_R & & & & \\
\hline & Frontal_Inf_Oper_R & & & & \\
\hline Cluster 10 & & 14 & -30 & 0 & 14 \\
\hline & Insula_L & & & & \\
\hline Cluster 11 & & 13 & 52 & 0 & -14 \\
\hline & Temporal_Pole_Sup_R & & & & \\
\hline & Temporal_Sup_R & & & & \\
\hline Cluster 12 & & 12 & 46 & 2 & -22 \\
\hline & Temporal_Pole_Sup_R & & & & \\
\hline & Temporal_Mid_R & & & & \\
\hline Cluster 13 & & 11 & -32 & -40 & 32 \\
\hline & White matter & & & & \\
\hline Cluster 14 & & 9 & 26 & -38 & 52 \\
\hline & Postcentral_R & & & & \\
\hline Cluster 15 & & 8 & 44 & 6 & -8 \\
\hline & Insula_R & & & & \\
\hline Cluster 16 & & 6 & -44 & -32 & -22 \\
\hline & Temporal_Inf_L & & & & \\
\hline Cluster 17 & & 6 & 6 & -64 & 62 \\
\hline & Precuneus_R & & & & \\
\hline
\end{tabular}

${ }^{*} R$ and $L$ stand for right and left. Mid, middle; Sup, superior; Inf, inferior; Orb, orbital; Oper, operculum; Cerebulum, cerebellum.

addiction tendency. In the following, we discuss our results at different scales of observation: (1) the crucial regions linking CIAS-R positive and CIAS-R negative networks, (2) regions with high proportions of connections related to internet addiction 

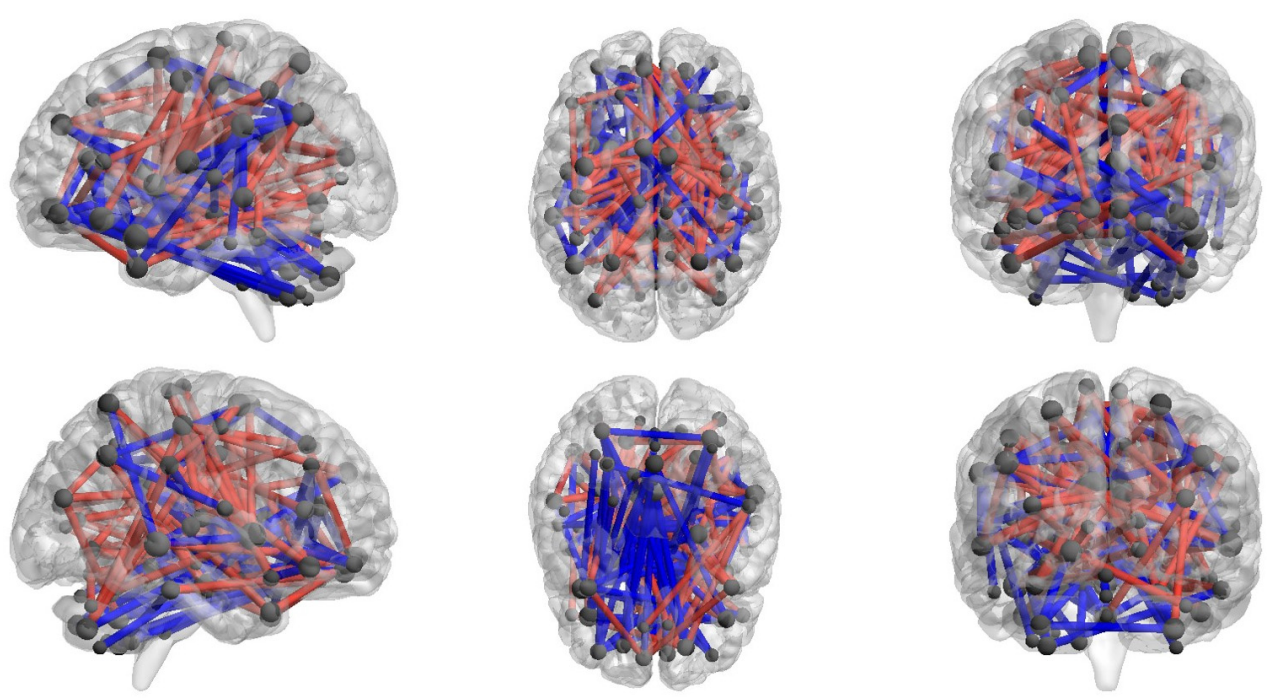

FIGURE 3 | Network of connections that are correlated with CIAS-R scores. Gray spheres represent the centroid of each node and are scaled accordingly to the number of significant edges they are associated with. Only nodes with connections are shown. Red lines represent edges that are positively associated with CIAS-R scores, while the blue lines represent edges that are negatively associated with CIAS-R scores.

tendency, and (3) the critical nodes altered by internet addiction tendency.

\section{Frontal Regions Link CIAS-R Positive and CIAS-R Negative Networks}

We observed that the majority of nodes that link the two (CIAS-R positive and CIAS-R negative) networks are located within the frontal lobe. These regions include the superior frontal gyrus, IFG, medial frontal gyrus, rolandic operculum, and supplementary motor area. The prefrontal cortex has been implicated to be a critical structure in cognitive control, inhibition, and response selection (Aron et al., 2004; Talati and Hirsch, 2005; Forstmann et al., 2008). Internet addiction is a phenomenon in that addicts have decreased self-control and decision making regarding internet usage, reflected by continued overuse despite their knowledge of negative effects. For example, several studies have found that participants with internet addiction showed higher fronto-striatal and frontoparietal activation during the Go/Nogo task (Ding et al., 2014; Ko et al., 2014; Chen et al., 2015) and Stroop task (Dong

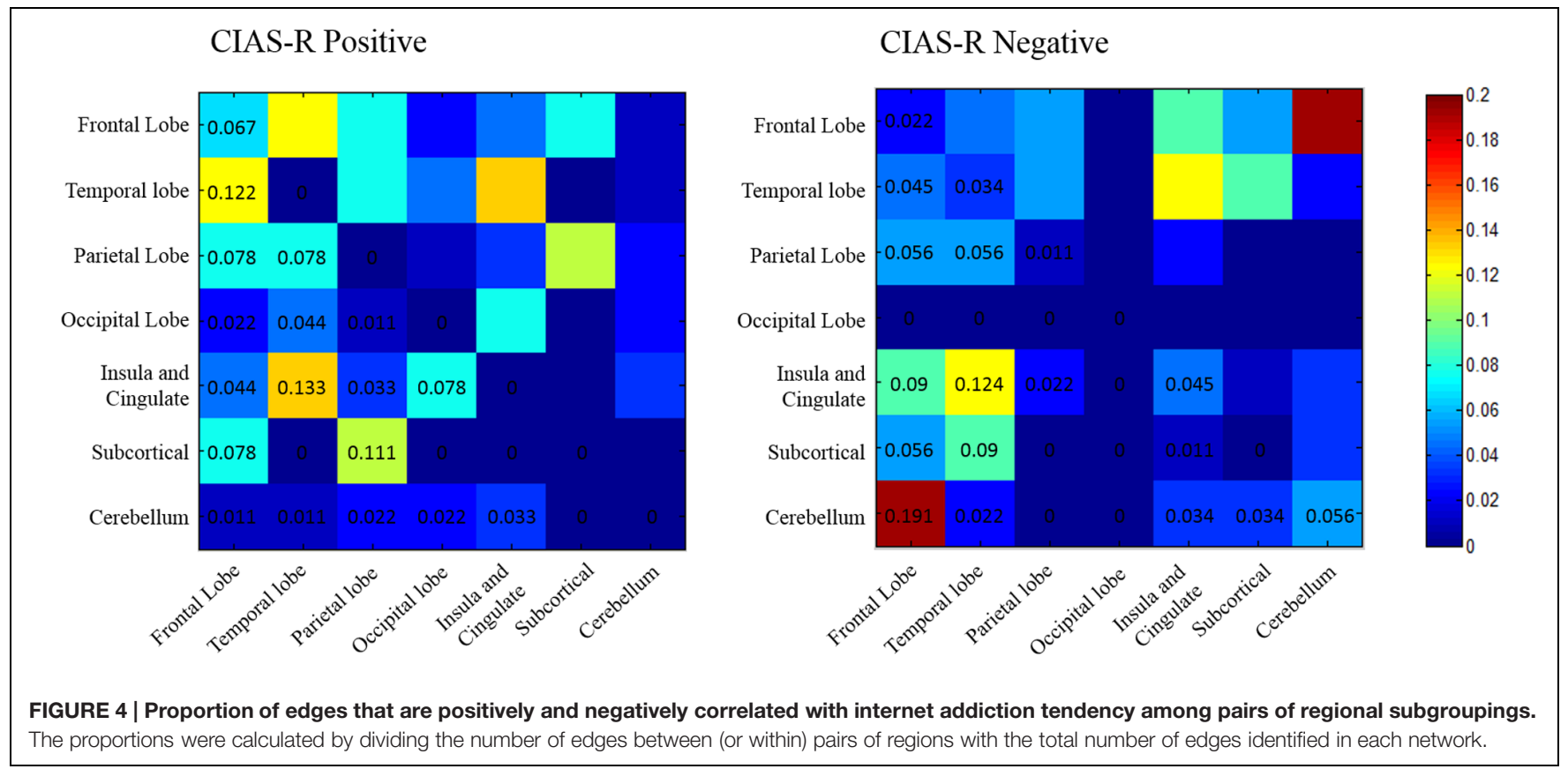


TABLE 3 | Node level analysis of internet addiction tendency.

\begin{tabular}{|c|c|c|c|c|c|c|}
\hline \multirow{2}{*}{$\begin{array}{l}\text { AAL Node } \\
\text { Cingulum_Post_L }\end{array}$} & \multicolumn{3}{|c|}{ MNI coordinate } & \multirow{2}{*}{$\begin{array}{c}\text { CIAS-R positive } \\
9\end{array}$} & \multirow{2}{*}{$\frac{\text { CIAS-R negative }}{8}$} & \multirow{2}{*}{$\frac{\text { Sum of correlated edges }}{17}$} \\
\hline & -4.85 & -42.92 & 24.67 & & & \\
\hline Cingulum_Post_R & 7.44 & -41.81 & 21.87 & 8 & 5 & 13 \\
\hline Insula_R & 39.02 & 6.25 & 2.08 & 7 & 6 & 13 \\
\hline Temporal_Mid_R & 57.47 & -37.23 & -1.47 & 5 & 4 & 9 \\
\hline Temporal_Pole_Sup_L & -39.88 & 15.14 & -20.18 & 4 & 5 & 9 \\
\hline Putamen_R & 27.78 & 4.91 & 2.46 & 5 & 3 & 8 \\
\hline Frontal_Inf_Orb_L & -35.98 & 30.71 & -12.11 & 1 & 7 & 8 \\
\hline
\end{tabular}

${ }^{*} R$ and $L$ stand for right and left. Post, posterior; Mid, middle; Sup, superior; Inf, inferior; Orb, orbital.

et al., 2012a, 2013, 2014), suggesting poorer response inhibition and error monitoring, and increased impulsivity. But on the other hand, internet addicts and video game players often show excellent performance of cognitive function, such as motor control and efficient decision making during gaming. Indeed, practice effects of video game play have been shown to generalize to a variety of enhanced executive skills, including perceptual, motor, attentional, and probabilistic inference skills (Green and Bavelier, 2003; Castel et al., 2005; Dye et al., 2009; Green et al., 2010; Green et al., 2012). One fMRI study found reduced recruitment of the fronto-parietal network in video game players compared to non-gamers during a high attentional demand task, possibly reflecting more efficient executive and attentional control (Bavelier et al., 2012). The two faces of cognitive control displayed by internet addicts poses an interesting dilemma. In our study, the observation of frontal regions linking the two networks where functional connectivity is decreased and increased by internet addiction tendency could reflect alterations in the frontal region for different aspects of cognitive control (i.e., for control of internet usage and gaming skills). It is worth mentioning that although Hong et al. (2013) hypothesized there could possibly be increased functional connectivity associated with practice effects in internet addicts, only decreased functional connectivity was observed in their study. One possibility proposed by Hong et al. (2013) for their absence of increased functional connectivity in internet-addicted individuals was that their small sample size resulted in the lack of power. By using seed-based analysis, which requires less multiple comparisons than whole-brain approaches, Hong et al. (2015) re-analyzed the 2013 data and observed both increased and decreased functional connectivity associated with internet addiction.

\section{The Widely Distributed Connections of the Internet Addiction Tendency Networks}

The data shows a large number of inter- and intra-hemispheric connections in both CIAS-R positive and CIAS- $\mathrm{R}$ negative networks, reflecting the extensive influence of internet addiction tendency on the brain. We observed that the highest proportion of connections in the CIAS-R positive network involved the "insula and cingulate - temporal," "frontal - temporal," and "subcortical - parietal" edges, while the highest proportion of connections in the CIAS-R negative network involved "frontal - cerebellar" and "insula and cingulate - temporal" edges
(Figure 4). In a recently proposed model of addiction (Moulton et al., 2014), the cerebellum helps maintain the homeostasis of the four interconnected circuits relevant to addiction: reward/saliency, motivation/drive, learning/memory as well as cognitive control. This model integrates the four-circuit model (Volkow et al., 2003, 2010) and the cerebellar functional resting state networks relating to executive and associative processing in the cerebral cortex (Buckner et al., 2011). The components for reward/saliency, motivation/drive, and learning/memory are amplified, while cognitive control is diminished in addiction. See Figure 6 for an illustration. Our observations of the highest functional connectivity proportions of the two internet addiction tendency networks are generally compatible with Moulton et al.'s (2014) model of the critical components involved in the addiction circuitry. Likewise, we did not observe many significant connections comprising the occipital lobe, which also dovetails Hong et al.'s (2013) findings. However, we additionally found a great proportion of "subcortical - parietal" edges that although is not particularly highlighted in the four-circuit model, these connections have been observed in the internet addiction literature (e.g., Ding et al., 2013; Hong et al., 2013, 2015), which could be due to a practice effect relating to internet usage.

\section{Critical Nodes Altered by Internet Addiction Tendency}

We identified nodes with the most connections are maximally related to internet addiction tendency. These nodes are those whose pattern of connections between the node itself and other brain regions are most susceptible to alteration by internet addiction tendency. The regions are specifically the bilateral posterior cingulate gyrus, right insula, right middle temporal gyrus, left superior temporal pole, right putamen, and the orbital part of left IFG (Figure 5). These regions have been implicated as key regions in many (internet) addiction studies and some have already been mentioned in the previous section. We now discuss the addiction literature highlighting these regions in more detail. The PCC, part of the default mode network and involved in various aspects of self-processing (Buckner et al., 2008; Fransson and Marrelec, 2008), served as a seed region in Ding et al.'s (2013) study, which showed significantly increased functional connectivity with the bilateral cerebellum posterior lobe and middle temporal gyrus, while decreased bilateral inferior parietal lobule and right inferior temporal gyrus in internet gaming addicts. Internet addicts have also been found to show 


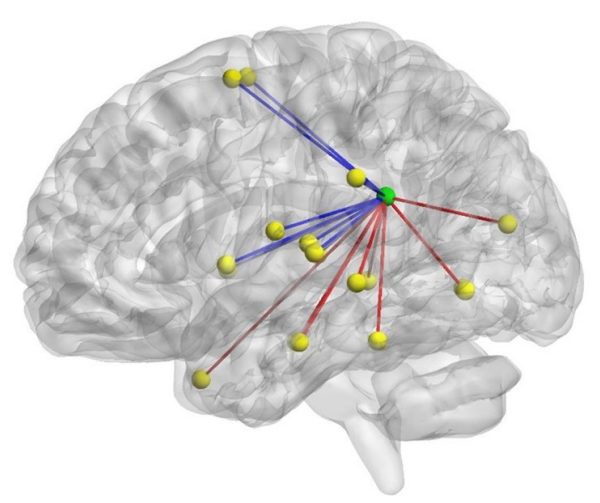

Cingulum_Post_L

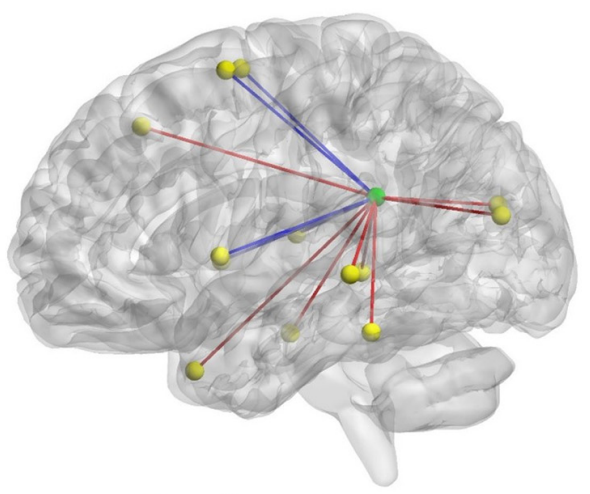

Cingulum_Post_R

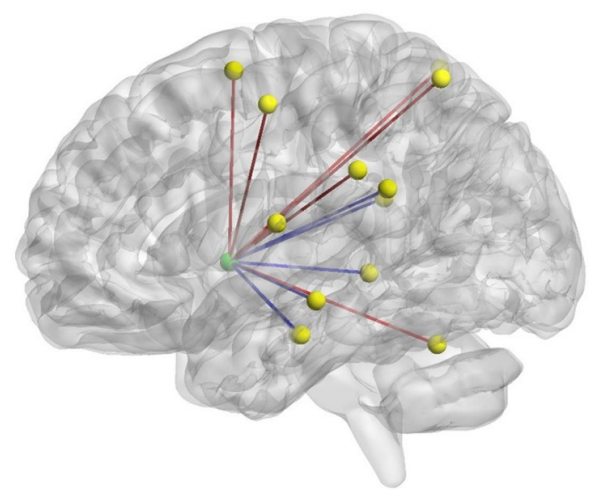

Insula

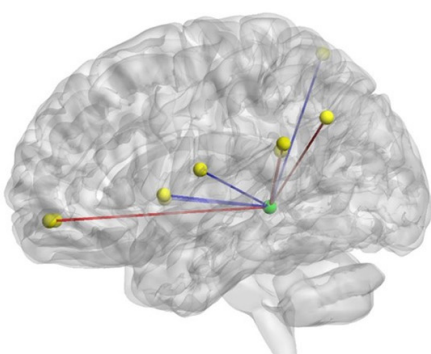

\section{Temporal_Mid_R}

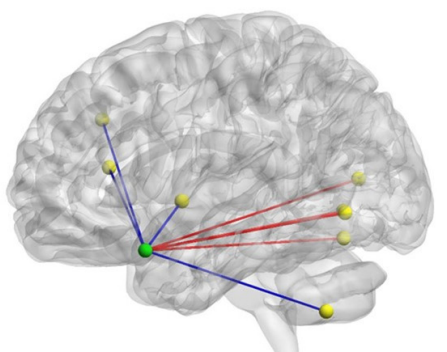

Temporal_Pole_Sup_L
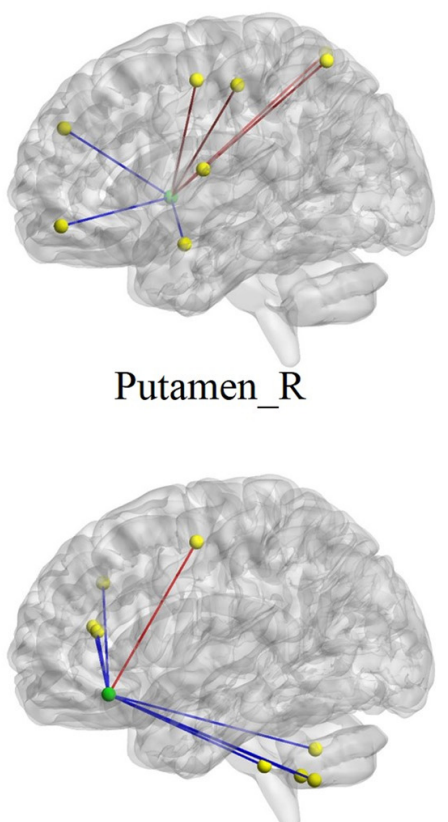

Frontal_Inf_Orb_L

FIGURE 5 | Visualization of the nodes with highest number of edges related to internet addiction tendency. Green spheres depict the centroid of each node with maximal edges, while yellow spheres depict their functional connectivity partners. Red lines indicate edges that are positively associated with CIAS-R scores, and blue lines represent edges that are negatively associated with CIAS-R scores. R and L stand for right and left. Post, posterior; Mid, middle; Sup, superior; Inf, inferior; Orb, orbital.

abnormal fractional anisotropy (Dong et al., 2012b) and gray matter density (Zhou et al., 2011) in the PCC. Zhang et al. (2015) chose the insula, which has been implicated in addiction (Naqvi and Bechara, 2009; Droutman et al., 2015), as the seed region and found altered functional connectivity with a network of regions in internet addicts. The role of the insula in addiction has been suggested for integrating interoceptive signals into conscious feelings (drug urges) and biases behavior during decision making (Naqvi and Bechara, 2009). The middle temporal gyrus and superior temporal pole has been observed in some internet 


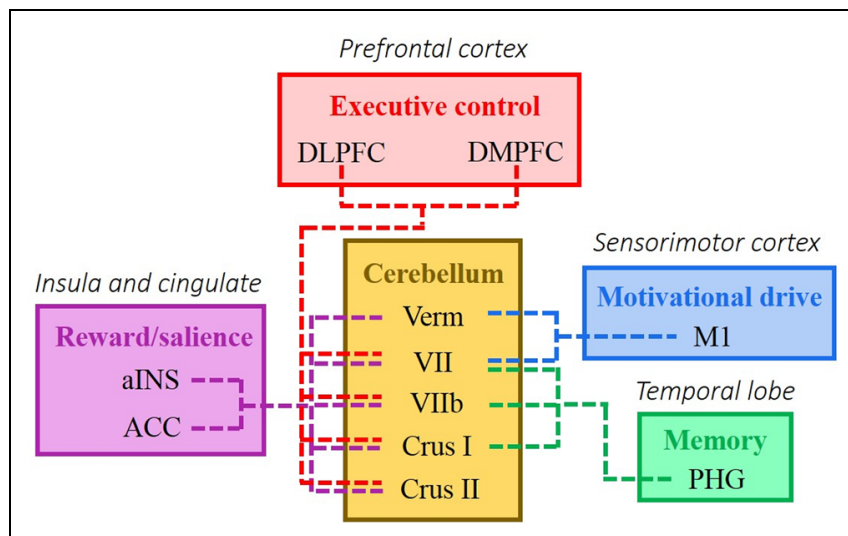

FIGURE 6 | A model of addiction highlighting the modulating role of the cerebellum of the four major brain networks proposed to be affected by addiction (adapted from Moulton et al., 2014). These circuits include reward/saliency, motivation/drive, learning/memory, and cognitive control. The colors correspond to different cerebral resting state networks: red, frontoparietal control network and default network; blue, early sensory cortices; green, dorsal attention; and purple, late motor cortices (Buckner et al., 2011; Yeo et al., 2011). ACC, anterior cingulate cortex; alNS, anterior insula; DLPFC, dorsolateral prefrontal cortex; DMPFC, dorsomedial prefrontal cortex; Hypo, hypothalamus; M1, primary motor cortex; PHG,

parahippocampal gyrus; SACC, subgenual anterior cingulate cortex; Verm, vermis; VI, cerebellar hemispheric lobule VI; VIlb, cerebellar hemispheric lobule VIllb.

addiction studies (see Meng et al., 2015 for a meta-analysis), and have been associated with game urge/craving, semantic processing, disembodiment, working memory, and emotional processing; however, their specific roles in addiction require further investigations. The putamen, part of the dorsal striatum, is also a critical region suggested by many addiction research (e.g., Ko et al., 2009; Ding et al., 2013; Lin et al., 2015), in which concomitant dopamine neurotransmission is involved in the development of compulsive drug-seeking and craving (Volkow et al., 2006; Koob and Volkow, 2010). Furthermore, research has suggested that dysfunction with the striato-thalamo-orbitofrontal circuit is a crucial cause of addiction, while the dorsal striatum involved in habit-learning and craving, the orbitofrontal cortex is involved with salience, drive, and compulsivity (Volkow and Fowler, 2000; Koob and Volkow, 2010; Volkow et al., 2010; Goldstein and Volkow, 2011). The abnormal functioning of the orbitofrontal cortex could explain the behavioral malfunctioning in addiction. Summarizing the above, the nodes we identified are hubs that are most susceptible to alteration by internet addiction tendency, and they have been identified repeatedly in the existing literature.

\section{Limitation}

As pointed out by one of our reviewers, whether to perform global signal regression in resting-state fMRI still remains a current debate. After re-analyzing the current data without global signal regression, our results turned out quite different compared to our original analysis and only $22.91 \%$ of the edges found in the NBS analyses without global signal regression overlapped with those of our current results. Without global signal regression, we did not find sufficient functional connections that were positively related to CIAS-R scores; however, we did find a network that comprised of functional connections that were negatively related to CIAS$\mathrm{R}$ scores. When identifying nodes with the most connections are maximally related to internet addiction tendency, we find consistency with the global signal regression analysis in that the cingulate, insula, temporal, and frontal areas were the most involved. However, several differences include the additional finding of bilateral supplementary motor areas and right angular gyrus showing decreased functional connectivity, and there were not as many subcortical regions in the identified network. While global signal regression still remains controversial, we decided to report both results. Details of the network identified without global signal regression is documented in the Supplementary Materials 3. Hopefully, future work on image preprocessing will shed light on which result is more accurate. At this moment, we suggest to interpret the current results with such caveats in mind.

\section{CONCLUSION}

Using a data-driven approach, we showed that network based statistics is a useful tool to characterize whole-brain connectivity affected by internet addiction tendency, identifying connections and critical regions that echo previous studies. Compared to seed analyses, this whole-brain approach provides a more comprehensive analysis of brain connections related to internet addiction, investigating a total of 6670 connections. We further showed that many functional connections and brain regions critical in clinical cases of addiction are also found to be associated with pre-clinical tendencies indexed by behavioral questionnaire measures. Although using a correlational approach, we cannot be sure whether these networks are altered as a result of internet usage or whether they are characteristics of people who are predisposed to higher risk of developing internet addiction, this research provides useful information in helping us understand the neural characteristics underlying addiction and its development.

\section{AUTHOR CONTRIBUTIONS}

TW performed the experiment, analyzed the data, interpreted the results, wrote and revised the manuscript. SH designed the experiment, wrote the grant proposal, guided the experiment's preparation and execution, helped in interpreting the data, preparing, and revising the manuscript.

\section{ACKNOWLEDGMENTS}

The authors are grateful to Yun-Ting Lee for help with data collection and Professor Po-Hsien Huang for statistical consultation. The study was funded by the Ministry of Science and Technology (MOST), Taiwan (MOST 102-2420-H-006-006-MY2 and MOST 104-2420-H-006-004MY2). In addition, this research was, in part, supported by the Ministry of Education (MoE), Taiwan, R.O.C. 
The Aim for the Top University Project to the National Cheng Kung University (NCKU). We thank the Mind Research and Imaging Center (MRIC), supported by the MOST, at NCKU for consultation and instrument availability. The CIAS-R questionnaire was provided by Sue-Huei Chen.

\section{REFERENCES}

American Psychiatric Association [APA] (2013). Diagnostic and Statistical Manual of Mental Disorders (DSM-5 $\left.{ }^{\circledR}\right)$. Arlington, VA: American Psychiatric Pub.

Aron, A. R., Robbins, T. W., and Poldrack, R. A. (2004). Inhibition and the right inferior frontal cortex. Trends Cogn. Sci. 8, 170-177. doi: 10.1016/j.tics.2004.02.010

Bavelier, D., Achtman, R. L., Mani, M., and Focker, J. (2012). Neural bases of selective attention in action video game players. Vis. Res. 61, 132-143. doi: 10.1016/j.visres.2011.08.007

Biswal, B. B., Mennes, M., Zuo, X. N., Gohel, S., Kelly, C., Smith, S. M., et al. (2010). Toward discovery science of human brain function. Proc. Natl. Acad. Sci. U.S.A. 107, 4734-4739. doi: 10.1073/pnas.0911855107

Brand, M., Young, K. S., and Laier, C. (2014). Prefrontal control and Internet addiction: a theoretical model and review of neuropsychological and neuroimaging findings. Front. Hum. Neurosci. 8:375. doi: 10.3389/fnhum.2014.00375

Buckner, R. L., Andrews-Hanna, J. R., and Schacter, D. L. (2008). The brain's default network - Anatomy, function, and relevance to disease. Year Cogn. Neurosci. 2008, 1-38. doi: 10.1196/annals.1440.011

Buckner, R. L., Krienen, F. M., Castellanos, A., Diaz, J. C., and Yeo, B. T. T. (2011). The organization of the human cerebellum estimated by intrinsic functional connectivity. J. Neurophysiol. 106, 2322-2345. doi: 10.1152/jn. 00339.2011

Castel, A. D., Pratt, J., and Drummond, E. (2005). The effects of action video game experience on the time course of inhibition of return and the efficiency of visual search. Acta Psychol. 119, 217-230. doi: 10.1016/j.actpsy.2005.02.004

Chen, C. Y., Huang, M. F., Yen, J. Y., Chen, C. S., Liu, G. C., Yen, C. F., et al. (2015). Brain correlates of response inhibition in Internet gaming disorder. Psychiatry Clin. Neurosci. 69, 201-209. doi: 10.1111/pcn.12224

Chen, S., Weng, L., Su, Y., Wu, H., and Yang, P. (2003). Development of a Chinese Internet addiction scale and its psychometric study. Chin. J. Psychol. 45, 251-266. doi: 10.1371/journal.pone.0098312

Craddock, R. C., Jbabdi, S., Yan, C. G., Vogelstein, J. T., Castellanos, F. X., Di Martino, A., et al. (2013). Imaging human connectomes at the macroscale. Nat. Methods 10, 524-539. doi: 10.1038/Nmeth.2482

Ding, W. N., Sun, J. H., Sun, Y. W., Chen, X., Zhou, Y., Zhuang, Z. G., et al. (2014). Trait impulsivity and impaired prefrontal impulse inhibition function in adolescents with internet gaming addiction revealed by a Go/No-Go fMRI study. Behav. Brain Funct. 10:20. doi: 10.1186/1744-9081-10-20

Ding, W. N., Sun, J. H., Sun, Y. W., Zhou, Y., Li, L., Xu, J. R., et al. (2013). Altered default network resting-state functional connectivity in adolescents with internet gaming addiction. PLOS ONE 8:e59902. doi: 10.1371/journal.pone.0059902

Dong, G. H., DeVito, E. E., Du, X. X., and Cui, Z. Y. (2012a). Impaired inhibitory control in 'internet addiction disorder': a functional magnetic resonance imaging study. Psychiatry Res. Neuroimaging 203, 153-158. doi: 10.1016/j.pscychresns.2012.02.001

Dong, G. H., DeVito, E., Huang, J., and Du, X. X. (2012b). Diffusion tensor imaging reveals thalamus and posterior cingulate cortex abnormalities in internet gaming addicts. J. Psychiatr. Res. 46, 1212-1216. doi: 10.1016/j.jpsychires.2012.05.015

Dong, G. H., Lin, X., Hu, Y. B., Xie, C. M., and Du, X. X. (2015a). Imbalanced functional link between executive control network and reward network explain the online-game seeking behaviors in Internet gaming disorder. Sci. Rep. 5:9197. doi: 10.1038/Srep09197

Dong, G. H., Lin, X., and Potenza, M. N. (2015b). Decreased functional connectivity in an executive control network is related to impaired executive function in Internet gaming disorder. Prog. Neuro-Psychopharmacol. Biol. Psychiatry 57, 76-85. doi: 10.1016/j.pnpbp.2014.10.012

\section{SUPPLEMENTARY MATERIAL}

The Supplementary Material for this article can be found online at: http://journal.frontiersin.org/article/10.3389/fnhum. 2016.00006

Dong, G. H., Lin, X., Zhou, H. L., and Lu, Q. L. (2014). Cognitive flexibility in internet addicts: fMRI evidence from difficult-to-easy and easy-to-difficult switching situations. Addict. Behav. 39, 677-683. doi: 10.1016/j.addbeh.2013.11.028

Dong, G. H., Shen, Y., Huang, J., and Du, X. X. (2013). Impaired error-monitoring function in people with internet addiction disorder: an event-related fMRI study. Eur. Addict. Res. 19, 269-275. doi: 10.1159/000346783

Droutman, V., Read, S. J., and Bechara, A. (2015). Revisiting the role of the insula in addiction. Trends Cogn. Sci. 19, 414-420. doi: 10.1016/j.tics.2015. 05.005

Dye, M. W. G., Green, C. S., and Bavelier, D. (2009). Increasing speed of processing with action video games. Curr. Dir. Psychol. Sci. 18, 321-326. doi: 10.1111/j.1467-8721.2009.01660.x

Finn, E. S., Shen, X., Holahan, J. M., Scheinost, D., Lacadie, C., Papademetris, X., et al. (2014). Disruption of functional networks in dyslexia: a wholebrain, data-driven analysis of connectivity. Biol. Psychiatry 76, 397-404. doi: 10.1016/j.biopsych.2013.08.031

Fornito, A., and Bullmore, E. T. (2015). Connectomics: a new paradigm for understanding brain disease. Eur. Neuropsychopharmacol. 25, 733-748. doi: 10.1016/j.euroneuro.2014.02.011

Fornito, A., Yoon, J., Zalesky, A., Bullmore, E. T., and Carter, C. S. (2011). General and specific functional connectivity disturbances in first-episode schizophrenia during cognitive control performance. Biol. Psychiatry 70, 64-72. doi: 10.1016/j.biopsych.2011.02.019

Forstmann, B. U., van den Wildenberg, W. P. M., and Ridderinkhof, K. R. (2008). Neural mechanisms, temporal dynamics, and individual differences in interference control. J. Cogn. Neurosci. 20, 1854-1865. doi: 10.1162/jocn.2008.20122

Fox, M. D., Zhang, D., Snyder, A. Z., and Raichle, M. E. (2009). The global signal and observed anticorrelated resting state brain networks. J. Neurophysiol. 101, 3270-3283. doi: 10.1152/jn.90777.2008

Fransson, P., and Marrelec, G. (2008). The precuneus/posterior cingulate cortex plays a pivotal role in the default mode network: evidence from a partial correlation network analysis. Neuroimage 42, 1178-1184. doi: 10.1016/j.neuroimage.2008.05.059

Goldstein, R. Z., and Volkow, N. D. (2011). Dysfunction of the prefrontal cortex in addiction: neuroimaging findings and clinical implications. Nat. Rev. Neurosci. 12, 652-669. doi: 10.1038/nrn3119

Green, C. S., and Bavelier, D. (2003). Action video game modifies visual selective attention. Nature 423, 534-537. doi: 10.1038/nature01647

Green, C. S., Pouget, A., and Bavelier, D. (2010). Improved probabilistic inference as a general learning mechanism with action video games. Curr. Biol. 20, 1573-1579. doi: 10.1016/j.cub.2010.07.040

Green, C. S., Sugarman, M. A., Medford, K., Klobusicky, E., and Bavelier, D. (2012). The effect of action video game experience on task-switching. Comput. Hum. Behav. 28, 984-994. doi: 10.1016/j.chb.2011.12.020

Griffiths, M. D., and Pontes, H. M. (2014). Internet addiction disorder and internet gaming disorder are not the same. J. Addict. Res. Ther. 5:e124. doi: 10.4172/2155-6105.1000e124

Han, C. E., Yoo, S. W., Seo, S. W., Na, D. L., and Seong, J. K. (2013). Cluster-based statistics for brain connectivity in correlation with behavioral measures. PLoS ONE 8:e72332. doi: 10.1371/journal.pone.0072332

Han, D. H., Kim, Y. S., Lee, Y. S., Min, K. J., and Renshaw, P. F. (2010). Changes in cue-induced, prefrontal cortex activity with video-game play. Cyberpsychol. Behav. Soc. Netw. 13, 655-661. doi: 10.1089/cyber.2009. 0327

Hoeft, F., Watson, C. L., Kesler, S. R., Bettinger, K. E., and Reiss, A. L. (2008). Gender differences in the mesocorticolimbic system during computer game-play. J. Psychiatr. Res. 42, 253-258. doi: 10.1016/j.jpsychires.2007. 11.010 
Hong, S. B., Harrison, B. J., Dandash, O., Choi, E. J., Kim, S. C., Kim, H. H., et al. (2015). A selective involvement of putamen functional connectivity in youth with internet gaming disorder. Brain Res. 1602, 85-95. doi: 10.1016/j.brainres.2014.12.042

Hong, S. B., Zalesky, A., Cocchi, L., Fornito, A., Choi, E. J., Kim, H. H., et al. (2013). Decreased functional brain connectivity in adolescents with internet addiction. PLoS ONE 8:e57831. doi: 10.1371/journal.pone.0057831

Király, O., Griffiths, M. D., Urbán, R., Farkas, J., Kökönyei, G., Elekes, Z., et al. (2014). Problematic internet use and problematic online gaming are not the same: findings from a large nationally representative adolescent sample. Cyberpsychol. Behav. Soc. Netw. 17, 749-754. doi: 10.1089/cyber.2014.0475

Ko, C. H., Hsieh, T. J., Chen, C. Y., Yen, C. F., Chen, C. S., Yen, J. Y., et al. (2014). Altered brain activation during response inhibition and error processing in subjects with internet gaming disorder: a functional magnetic imaging study. Eur. Arch. Psychiatry Clin. Neurosci. 264, 661-672. doi: 10.1007/s00406-0130483-3

Ko, C. H., Hsieh, T. J., Wang, P. W., Lin, W. C., Yen, C. F., Chen, C. S., et al. (2015). Altered gray matter density and disrupted functional connectivity of the amygdala in adults with Internet gaming disorder. Prog. Neuro-Psychopharmacol. Biol. Psychiatry 57, 185-192. doi: 10.1016/j.pnpbp.2014.11.003

Ko, C. H., Liu, G. C., Hsiao, S. M., Yen, J. Y., Yang, M. J., Lin, W. C., et al. (2009). Brain activities associated with gaming urge of online gaming addiction. J. Psychiatr. Res. 43, 739-747. doi: 10.1016/j.jpsychires.2008.09.012

Ko, C. H., Liu, G. C., Yen, J. Y., Chen, C. Y., Yen, C. F., and Chen, C. S. (2013). Brain correlates of craving for online gaming under cue exposure in subjects with Internet gaming addiction and in remitted subjects. Addict. Biol. 18, 559-569. doi: 10.1111/j.1369-1600.2011.00405.X

Ko, C.-H., Yen, C.-F., Yen, C.-N., Yen, J.-Y., Chen, C.-C., and Chen, S.-H. (2005). Screening for internet addiction: an empirical study on cut-off points for the chen internet addiction scale. Kaohsiung J. Med. Sci. 21, 545-551. doi: 10.1016/S1607-551X(09)70206-2

Kong X.-Z., Zhen, Z., Li, X., Lu, H.-H., Wang, R., Liu, L., et al. (2014). Individual differences in impulsivity predict head motion during magnetic resonance imaging. PLoS ONE 9:e104989. doi: 10.1371/journal.pone.0104989

Koob, G. F., and Volkow, N. D. (2010). Neurocircuitry of addiction. Neuropsychopharmacology 35, 217-238. doi: 10.1038/npp.2009.110

Kühn, S., and Gallinat, J. (2015). Brains online: structural and functional correlates of habitual Internet use. Addict. Biol. 20, 415-422. doi: 10.1111/adb.12128

Kuss, D. J., and Griffiths, M. D. (2012). Internet gaming addiction: a systematic review of empirical research. Int. J. Mental Health Addict. 10, 278-296. doi: 10.1007/s11469-011-9318-5

Li, B. J., Friston, K. J., Liu, J., Liu, Y., Zhang, G. P., Cao, F. L., et al. (2014). Impaired frontal-basal ganglia connectivity in adolescents with internet addiction. Sci. Rep. 4:5027. doi: 10.1038/Srep05027

Li, W. W., Li, Y. D., Yang, W. J., Zhang, Q. L., Wei, D. T., Li, W. F., et al. (2015). Brain structures and functional connectivity associated with individual differences in Internet tendency in healthy young adults. Neuropsychologia 70, 134-144. doi: 10.1016/j.neuropsychologia.2015.02.019

Lin, F. C., Zhou, Y., Du, Y. S., Zhao, Z. M., Qin, L. D., Xu, J. R., et al. (2015). Aberrant corticostriatal functional circuits in adolescents with Internet addiction disorder. Front. Hum. Neurosci. 9:356. doi: 10.3389/fnhum.2015.00356

Lin, M. P., Ko, H. C., and Wu, J. Y. W. (2011). Prevalence and psychosocial risk factors associated with internet addiction in a nationally representative sample of college students in taiwan. Cyberpsychol. Behav. Soci. Netw. 14, 741-746. doi: $10.1089 /$ cyber.2010.0574

Lorenz, R. C., Kruger, J. K., Neumann, B., Schott, B. H., Kaufmann, C., Heinz, A., et al. (2013). Cue reactivity and its inhibition in pathological computer game players. Addict. Biol. 18, 134-146. doi: 10.1111/j.1369-1600.2012. 00491.x

Meng, Y. J., Deng, W., Wang, H. Y., Guo, W. J., and Li, T. (2015). The prefrontal dysfunction in individuals with Internet gaming disorder: a meta-analysis of functional magnetic resonance imaging studies. Addict. Biol. 20, 799-808. doi: 10.1111/adb. 12154

Moulton, E. A., Elman, I., Becerra, L. R., Goldstein, R. Z., and Borsook, D. (2014). The cerebellum and addiction: insights gained from neuroimaging research. Addict. Biol. 19, 317-331. doi: 10.1111/adb.12101
Naqvi, N. H., and Bechara, A. (2009). The hidden island of addiction: the insula. Trends Neurosci. 32, 56-67. doi: 10.1016/j.tins.2008.09.009

Nichols, T. E., and Holmes, A. P. (2002). Nonparametric permutation tests for functional neuroimaging: a primer with examples. Hum. Brain Mapp. 15, 1-25. doi: $10.1002 / \mathrm{hbm} .1058$

OReilly, M. (1996). Internet addiction: a new disorder enters the medical lexicon. Can. Med. Associ. J. 154, 1882-1883.

Park, C. H., Chun, J. W., Cho, H., Jung, Y. C., Choi, J., and Kim, D. J. (2015). Is the internet gaming-addicted brain close to be in a pathological state? Addict. Biol. doi: $10.1111 / \mathrm{adb} .12282$ [Epub ahead of print].

Petry, N. M., and O'Brien, C. P. (2013). Internet gaming disorder and the DSM-5. Addiction 108, 1186-1187. doi: 10.1111/add.12162

Petry, N. M., Rehbein, F., Gentile, D. A., Lemmens, J. S., Rumpf, H. J., Mößle, T., et al. (2014). An international consensus for assessing internet gaming disorder using the new DSM-5 approach. Addiction 109, 1399-1406. doi: 10.1111 /add. 12457

Rorden, C., Karnath, H. O., and Bonilha, L. (2007). Improving lesion-symptom mapping. J. Cogn. Neurosci. 19, 1081-1088. doi: 10.1162/jocn.2007.19.7.1081

Saad, Z. S., Gotts, S. J., Murphy, K., Chen, G., Jo, H. J., Martin, A., et al. (2012). Trouble at rest: how correlation patterns and group differences become distorted after global signal regression. Brain Connect. 2, 25-32. doi: 10.1089/brain.2012.0080

Song, X. W., Dong, Z. Y., Long, X. Y., Li, S. F., Zuo, X. N., Zhu, C. Z., et al. (2011). REST: a toolkit for resting-state functional magnetic resonance imaging data processing. PLoS ONE 6:e25031. doi: 10.1371/journal.pone.0025031

Stanley, M. L., Moussa, M. N., Paolini, B. M., Lyday, R. G., Burdette, J. H., and Laurienti, P. J. (2013). Defining nodes in complex brain networks. Front. Comput. Neurosci. 7:169. doi: 10.3389/fncom.2013.00169

Sun, Y. J., Ying, H., Seetohul, R. M., Wang, X. M., Ya, Z., Qian, L., et al. (2012). Brain fMRI study of crave induced by cue pictures in online game addicts (male adolescents). Behav. Brain Res. 233, 563-576. doi: 10.1016/j.bbr.2012.05.005

Takeuchi, H., Taki, Y., Nouchi, R., Sekiguchi, A., Hashizume, H., Sassa, Y., et al. (2014). Association between resting-state functional connectivity and empathizing/systemizing. Neuroimage 99, 312-322. doi: 10.1016/j.neuroimage.2014.05.031

Talati, A., and Hirsch, J. (2005). Functional specialization within the medial frontal gyrus for perceptual go/no-go decisions based on "what," "when," and "where" related information: an fMRI study. J. Cogn. Neurosci. 17, 981-993. doi: 10.1162/0898929054475226

Tzourio-Mazoyer, N., Landeau, B., Papathanassiou, D., Crivello, F., Etard, O., Delcroix, N., et al. (2002). Automated anatomical labeling of activations in SPM using a macroscopic anatomical parcellation of the MNI MRI single-subject brain. Neuroimage 15, 273-289. doi: 10.1006/nimg.2001.0978

van den Heuvel, M. P., and Pol, H. E. H. (2010). Exploring the brain network: a review on resting-state fMRI functional connectivity. Eur. Neuropsychopharmacol. 20, 519-534. doi: 10.1016/j.euroneuro.2010.03.008

Volkow, N. D., and Fowler, J. S. (2000). Addiction, a disease of compulsion and drive: involvement of the orbitofrontal cortex. Cereb. Cortex 10, 318-325. doi: $10.1093 /$ cercor/10.3.318

Volkow, N. D., Fowler, J. S., and Wang, G. J. (2003). The addicted human brain: insights from imaging studies. J. Clin. Investig. 111, 1444-1451. doi: $10.1172 /$ Jci200318533

Volkow, N. D., Wang, G. J., Fowler, J. S., Tomasi, D., Telang, F., and Baler, R. (2010). Addiction: decreased reward sensitivity and increased expectation sensitivity conspire to overwhelm the brain's control circuit. Bioessays 32, 748-755. doi: 10.1002/bies.201000042

Volkow, N. D., Wang, G. J., Telang, F., Fowler, J. S., Logan, J., Childress, A. R., et al. (2006). Cocaine cues and dopamine in dorsal striatum: mechanism of craving in cocaine addiction. J. Neurosci. 26, 6583-6588. doi: 10.1523/Jneurosci.154406.2006

Wang, Y., Yin, Y., Sun, Y. W., Zhou, Y., Chen, X., Ding, W. N., et al. (2015). Decreased prefrontal lobe interhemispheric functional connectivity in adolescents with internet gaming disorder: a primary study using resting-state fMRI. PLoS ONE 10:e0118733. doi: 10.1371/journal.pone.0118733

Wee, C. Y., Zhao, Z. M., Yap, P. T., Wu, G. R., Shi, F., Price, T., et al. (2014). Disrupted brain functional network in internet addiction disorder: a restingstate functional magnetic resonance imaging study. PLOS ONE 9:e107306. doi: 10.1371/journal.pone.0107306 
Weissenbacher, A., Kasess, C., Gerstl, F., Lanzenberger, R., Moser, E., and Windischberger, C. (2009). Correlations and anticorrelations in resting-state functional connectivity MRI: a quantitative comparison of preprocessing strategies. Neuroimage 47, 1408-1416. doi: 10.1016/j.neuroimage.2009. 05.005

Xia, M., Wang, J., and He, Y. (2013). BrainNet Viewer: a network visualization tool for human brain connectomics. PLoS ONE 8:e68910. doi: 10.1371/journal.pone.0068910

Yan, C., and Zang, Y. (2010). DPARSF: a MATLAB toolbox for "pipeline" data analysis of resting-state fMRI. Front. Syst. Neurosci. 4:13. doi: $10.3389 /$ fnsys.2010.00013

Yarkoni, T., Poldrack, R. A., Nichols, T. E., Van Essen, D. C., and Wager, T. D. (2011). Large-scale automated synthesis of human functional neuroimaging data. Nat. Methods 8, 665-670. doi: 10.1038/nmeth.1635

Yeo, B. T. T., Krienen, F. M., Sepulcre, J., Sabuncu, M. R., Lashkari, D., Hollinshead, M., et al. (2011). The organization of the human cerebral cortex estimated by intrinsic functional connectivity. J. Neurophysiol. 106, 1125-1165. doi: $10.1152 /$ jn.00338.2011

Young, K. S. (1998). Internet addiction: the emergence of a new clinical disorder. CyberPsychol. Behav. 1, 237-244. doi: 10.1007/s10899-011-9287-4

Yuan, K., Qin, W., Yu, D., Bi, Y., Xing, L., Jin, C., et al. (2015). Core brain networks interactions and cognitive control in internet gaming disorder individuals in late adolescence/early adulthood. Brain Struct. Funct. doi: 10.1007/s00429-0140982-7 [Epub ahead of print].
Zalesky, A., Fornito, A., and Bullmore, E. T. (2010a). Network-based statistic: identifying differences in brain networks. Neuroimage 53, 1197-1207. doi: 10.1016/j.neuroimage.2010.06.041

Zalesky, A., Fornito, A., Harding, I. H., Cocchi, L., Yücel, M., Pantelis, C., et al. (2010b). Whole-brain anatomical networks: does the choice of nodes matter? Neuroimage 50, 970-983. doi: 10.1016/j.neuroimage.2009.12.027

Zhang, J. T., Yao, Y. W., Li, C. S. R., Zang, Y. F., Shen, Z. J., Liu, L., et al. (2015). Altered resting-state functional connectivity of the insula in young adults with Internet gaming disorder. Addict. Biol. doi: 10.1111/adb.12247 [Epub ahead of print].

Zhou, Y., Lin, F. C., Du, Y. S., Qin, L. D., Zhao, Z. M., Xu, J. R., et al. (2011). Gray matter abnormalities in Internet addiction: a voxel-based morphometry study. Eur. J. Radiol. 79, 92-95. doi: 10.1016/j.ejrad.2009.10.025

Conflict of Interest Statement: The authors declare that the research was conducted in the absence of any commercial or financial relationships that could be construed as a potential conflict of interest.

Copyright $\odot 2016$ Wen and Hsieh. This is an open-access article distributed under the terms of the Creative Commons Attribution License (CC BY). The use, distribution or reproduction in other forums is permitted, provided the original author(s) or licensor are credited and that the original publication in this journal is cited, in accordance with accepted academic practice. No use, distribution or reproduction is permitted which does not comply with these terms. 